\title{
Personalidade hardiness e fatores associados em profissionais da saúde atuantes em serviços que atendem pacientes críticos
}

\author{
Hardy personality and associated factors in health professionals \\ active in services that treat critical patients
}

Renê Ferreira da Silva-Junior (https://orcid.org/0000-0002-3462-3930) ${ }^{1}$

Elaine Cristina Santos Alves (https://orcid.org/0000-0003-0691-9969) ${ }^{1}$

Kamilla de Oliveira Santos (https://orcid.org/0000-0002-0008-1387) ${ }^{1}$

Silvânia Paiva dos Santos (https://orcid.org/0000-0002-6911-8256) ${ }^{1}$

Henrique Andrade Barbosa (https://orcid.org/0000-0001-8650-8064) ${ }^{1}$

Leila das Graças Siqueira (https://orcid.org/0000-0002-1538-6722) ${ }^{1}$

Jaqueline D' Paula Ribeiro Vieira Torres (https://orcid.org/0000-0003-2383-2523) ${ }^{1}$

Carla Silvana Oliveira e Silva (https://orcid.org/0000-0002-2752-1557) ${ }^{1}$

${ }^{1}$ Centro de Ciências

Biológicas e da Saúde,

Universidade Estadual de

Montes Claros. R. Ruy Braga

s/n, Vila Mauricéia. 39400-

000 Montes Claros MG

Brasil.renejunior_deny@

hotmail.com

\begin{abstract}
This study aimed to identify factors associated with the hardy personality in health professionals working in hospital services that treat critically ill patients. This is an epidemiological, cross-sectional, and analytical study conducted with 469 health professionals. We used the Hardiness Scale (HS), coupled with a questionnaire to investigate sociodemographic, occupational, and health conditions. A descriptive and bivariate analysis was performed using the chi-square test and the multiple model through multiple logistic regression, using the Hosmer-Lemeshow and PseudoR ${ }^{2}$ tests, estimating odds ratios. The classification of total scores in HS showed prevailing moderate hardiness (48.4\%). Professionals with a fair or poor fantastic lifestyle (FL) were $74 \%$ less likely to show high hardiness than those with very good and excellent FL. Also, professionals who were not on leave, who had high satisfaction with compassion at work, low stress, and low burnout were more likely to show high hardiness. The har$d y$ personality, directly and indirectly, influences the health and well-being of health professionals working in hospital services that treat critically ill patients.
\end{abstract}

Key words Psychological resilience, Health professionals, Worker's health
Resumo Objetivou-se identificar os fatores associados à personalidade hardiness em profissionais de saúde atuantes em serviços hospitalares que atendem a pacientes críticos. Estudo epidemiológico, transversal e analítico, realizado com 469 profissionais de saúde. Como instrumento, utilizou-se a Escala Hardiness (EH) associada a um questionário para investigar as condições sociodemográficas, ocupacionais e de saúde. Foi realizada a análise descritiva e bivariada por meio do teste qui-quadrado e modelo múltiplo através de regressão logística múltipla, utilizando o teste Hosmer Lemeshow e PseudoR², estimando razão das chances (odds ratio). A classificação dos escores totais na EH evidenciou preponderância de moderado hardiness (48,4\%). A chance de alto hardiness entre profissionais com estilo de vida fantástico regular ou ruim reduziu em $74 \%$ quando comparado aos considerados muito bons e excelentes. Também esteve aumentada entre os profissionais que não tiveram licença/afastamento, que apresentavam alta satisfação por compaixão no trabalho, baixo estresse e baixo burnout. $A$ personalidade hardiness influência direta e indiretamente a saúde e o bem-estar do profissional de saúde atuante em serviços hospitalares que atendem a pacientes críticos.

Palavras-chave Resiliência psicológica, Profissionais de saúde, Saúde do trabalhador 


\section{Introdução}

O estresse ocupacional é um fenômeno mundial com elevado impacto nas organizações, principalmente nos serviços de saúde, pois compromete tanto a qualidade de vida dos profissionais como a segurança da assistência prestada ao paciente $^{1,2}$.

Situações estressoras, em profissionais de saúde da área hospitalar, tais como alta carga horária, sobrecarga de tarefas e situações-limite de vida e morte podem ocorrer durante um considerável período de tempo sobre um indivíduo que não possua condição psicológica para se esquivar ou controlá-las, podendo resultar em alterações fisiológicas, problemas emocionais e outros sintomas, vindo a desenvolver o estresse ${ }^{3}$.

Por outro lado, o desenvolvimento de resistência e estratégias de enfrentamento são mecanismos para reduzir ou neutralizar estressores entre os profissionais de saúde, sendo que, dentre os vários recursos internos que os seres humanos possuem, a resistência tem sido sugerida como uma solução para superar as várias situações adversas que possam surgir ${ }^{4}$.

Desta maneira, as características ou traços de personalidade têm sido estudados, com o intuito de identificar indivíduos que podem ter maior risco para o desenvolvimento de estresse ou que sejam refratários. Destacam-se as pesquisas sobre hardiness, cujo conceito começou a ser formulado na década de 1970, nos Estados Unidos, sendo definido pela presença de traços de personalidade ou características individuais que possibilitam a resistência aos estressores ${ }^{1}$. A personalidade hardiness ou hardy refere-se, portanto, a um recurso pessoal contra os efeitos de acontecimentos negativos ou estressantes sobre a saúde dos profissionais ${ }^{4-6}$.

Desse modo, conceitos como rusticidade, resistência psicológica, personalidade resistente, resiliência ou resistência ao estresse são utilizados para a representação do constructo da personalidade $h a r d y$, sendo que a sua essência se determina por três bases conceituais: o modelo de diferenças individuais nas respostas ao estresse; $\mathrm{o}$ modelo de Lazarus (1966) sobre os mecanismos de avaliação de tensão e as contribuições dos autores acerca de benefícios de algumas disposições de personalidade no processo de estresse $\mathrm{e}^{5-7}$.

A personalidade hardiness engloba conceitos em três domínios: controle; compromisso e desafio, envolvendo a crença de poder controlar ou influenciar os eventos de sua experiência, a habilidade de sentir-se completamente envolvido ou comprometido nas atividades de sua vida e a antecipação da mudança como um desafio excitante para o crescimento pessoal, respectivamente ${ }^{8}$.

Fatores associados a elevados níveis de resistência são mais frequentes em indivíduos que manifestam felicidade, satisfação no trabalho, satisfação com a vida, boa saúde física e mental, autoconfiança, autoconsciência, autogerenciamento e motivação em melhorar os resultados, enquanto os que possuem baixa resistência são mais susceptíveis a apresentarem depressão, ansiedade e doenças cardiovasculares e neuroendócrinas $^{5,9-11}$.

Diante deste cenário e devido à importância do desenvolvimento da resistência ocupacional, a personalidade hardiness apresenta-se como um recurso que pode ser desenvolvido permanecendo razoavelmente estável ao longo do tempo. Treinamentos para aumento da resistência podem ser eficazes, existindo, na atualidade, uma grande indústria construída em torno de ensino da resistência ${ }^{9-11}$.

Investigar as variáveis que influenciam na resistência ao estresse laboral é importante, visto que os estressores têm efeitos deletérios sobre o profissional e que a melhor resistência psicológica de um indivíduo pode aumentar a sua capacidade de lidar com as adversidades laborais ${ }^{11,12}$. Assim, o objetivo deste estudo foi identificar os fatores associados à personalidade hardiness em profissionais de saúde atuantes em serviço hospitalares que atendem pacientes críticos no Norte de Minas Gerais/Brasil.

\section{Materiais e métodos}

Estudo epidemiológico, transversal e analítico, desenvolvido nos serviços de saúde da macrorregião norte de Minas Gerais/Brasil, que é composta por 86 municípios e referência para uma população de 1.670.268 habitantes ${ }^{13}$. Fizeram parte da pesquisa profissionais de saúde (enfermeiros, fisioterapeutas, nutricionais, médicos e técnicos de enfermagem) que prestavam atendimento direto ao paciente, atuantes em setores que atendem pacientes críticos: setor de oncologia, nefrologia, terapia intensiva neonatal e pronto socorro, das cidades de Montes Claros, Pirapora, Janaúba, Brasília de Minas e Salinas.

Para compor o tamanho da amostra realizouse uma seleção onde se permitiu que fossem incluídos somente profissionais com mais de seis meses de trabalho no setor e que aceitaram participar do estudo, e excluídos aqueles em afasta- 
mento das atividades laborais ou em período de férias no momento da coleta de dados, além daqueles que se recusaram a participar da pesquisa, não tendo assinado o termo de consentimento livre e esclarecido.

Após o levantamento em todos os serviços que atenderam aos critérios de inclusão, o número total de profissionais atuantes em tais serviços no período da coleta de dados foi de 910 sujeitos. E, para o cálculo amostral utilizou-se a amostra aleatória simples com reposição. A seleção ocorreu por meio de sorteio utilizando-se o programa Excel for Windows ${ }^{\circledR}$. Para estimar o tamanho da amostra, optou-se por um erro amostral tolerável de 5\%, intervalo de confiança de $95 \%$, prevalência para o evento de $50 \%$, considerando $20 \%$ de possíveis perdas, totalizando 450 indivíduos; assim, a amostra se constituiu de 469 profissionais de saúde incluídos no estudo.

A coleta de dados foi realizada no período de maio 2017 a abril de 2018. A variável desfecho personalidade hardiness foi avaliada através da aplicação da Escala Hardiness (EH), que tem por finalidade avaliar o quanto de atitudes hardy os indivíduos possuem no enfrentamento de situações estressantes. A EH apresenta-se adaptada para a língua portuguesa do Brasil, com consistência interna satisfatória e validade de construto na população estudada e é de livre domínio e propriedade intelectual registrada. É uma escala tipo likert, auto-aplicável, com 30 itens, com respostas que variam de zero (nada verdadeiro) a três (completamente verdadeiro) ${ }^{12}$.

O resultado é obtido por meio da soma dos itens, tendo os escores das questões $3,4,5,6,8$, $13,16,18,19,20,22,23,25,28$ e 30 invertidos, para, então, serem somados e permitindo o resultado pela composição total da escala e pelos três domínios: Compromisso $(1,6,7,11,16,17$, 22, 27, 28 e 30), Controle (2, 3, 8, 9, 12, 15, 18, 20 , 25 e 29) e Desafio $(4,5,10,13,14,19,21,23,24$ e 26). A pontuação da escala pode variar de 0 a 90 para a composição total da escala; e de 0 a 30 para a composição dos domínios, sendo classificado como baixo hardiness o indivíduo que apresentar percentil < 25\%; moderado entre $25 \%$ e $75 \%$ e alto hardiness se o percentil $>75 \%{ }^{14}$.

Para identificar os fatores associados à hardiness, utilizou-se questionários autoaplicáveis, os quais foram entregues aos participantes e posteriormente, recolhidos por pesquisadores devidamente treinados. Os instrumentos foram compostos com questões referentes às características sociodemográficas; ambiente de trabalho; comportamentais (Estilo de Vida Fantástico (EVF); saúde física e relacionada à saúde mental, através da Escala de Estresse no Trabalho (EET); Maslach Burnout Inventory-Human Services Survey (MBI -HSS); inventário de ansiedade de Beck (BAI); Inventário de Depressão de Beck (BDI), qualidade de vida no trabalho (ProQOL) e escala de desesperança de Beck (BHS) ${ }^{15-20}$.

Os dados foram tabulados por meio do software Statistical Package Social Science (SPSS), versão 20.0. Para a análise dos dados, foi realizada análise descritiva de todas as variáveis por meio de sua distribuição de frequência absoluta (n) e relativa (\%). $\mathrm{Na}$ análise bivariada, foi aplicado o teste qui-quadrado para verificar a associação entre a variável dependente e as variáveis independentes ao nível de $\mathrm{p}<0,20$. As variáveis que apresentaram valor de $\mathrm{p}<0,20$ foram selecionadas para o modelo múltiplo, adotando um modelo de regressão logística. Para a análise da qualidade do ajuste do modelo foi utilizado o teste Hosmer Lemeshow e PseudoR ${ }^{2}$ estimando razão das chances (odds ratio), com seus respectivos intervalos de confiança de 95\% (IC 95\%). Nesta etapa múltipla, as variáveis que apresentaram $\mathrm{p} \leq 0,05$ foram mantidas no modelo final.

O estudo atendeu aos princípios éticos propostos na resolução 466/12 e foi aprovado pelo Comitê de Ética em pesquisa da Universidade Estadual de Montes Claros - UNIMONTES.

\section{Resultados}

Participaram deste estudo 469 profissionais de saúde. $\mathrm{Na}$ análise descritiva univariada, observou-se que dentre os dados sociodemográficos, $65,7 \%$ dos participantes eram do sexo feminino, com idade entre 30-39 anos $(48,8 \%)$, casados ou união estável (61,8\%); e possuíam filhos $(64,4 \%)$. $69,1 \%$ eram católicos, com renda de 3 a 6 salários mínimos (40,9\%).

Em relação às características do trabalho, $31,6 \%$ trabalhavam no setor de nefrologia e $31,6 \%$ no pronto socorro; $52,7 \%$ atuavam escala de plantão de 12 horas, com 36 horas de descanso e 73,1\% possuíam carga horária semanal de 41 a 80 horas. $54,2 \%$ trabalhavam no turno diurno, com tempo de atuação na área de 10 a 15 anos (34,3\%); possuíam tempo de atuação no setor maior ou igual a 5 anos (53,1\%), e 63,1\% atuavam em apenas um emprego. A maioria dos participantes apresentava vínculo de trabalho como contratado/celetista (67,0\%). Em relação à profissão, 66,3\% eram técnicos de enfermagem, 15,6\% enfermeiros, $8,1 \%$ médicos e $10 \%$ outros profissionais. 
Quanto à saúde mental e física, $89,3 \%$ dos profissionais apresentaram ausência de ansiedade, $97,2 \%$ demonstraram depressão ausente/leve e desesperança moderada/leve e 95,9\% apresentaram depressão baixa/média. 51,8\% referiram alta satisfação por compaixão; $63,3 \%$ apresentaram estresse traumático secundário e 73,3\% demonstraram baixos escores para burnout. Em relação à aparência física consideraram-se muito satisfeitos $(59,7 \%)$, e a maioria dos entrevistados considerou como bom o seu estado de saúde $(64,1 \%)$, além de referir não estar em uso de medicação para dormir ou de uso contínuo $(81,4 \%)$, também apresentaram classificação média de estresse no trabalho( $(48,8 \%)$.

No que tange ao comportamento, o EVF mostrou-se muito bom para $52,2 \%$ dos profissionais; $64,6 \%$ não eram fisicamente ativos ( $<3$ vezes/ semana), 57,6\% relataram uma dieta balanceada, $70,6 \%$ se consideravam fora do peso, $97,4 \%$ não tabagistas, nenhum declarou etilista e $55,9 \%$ possuíam bom sono.

Em relação à Classificação dos escores total da EH, 27,29\% dos profissionais de saúde pesquisados apresentaram alto hardiness, $48,4 \%$ moderado e $24,3 \%$ baixo.

Nas Tabelas 1, 2 e 3 foi realizada a análise bivariada para verificar a associação da variável desfecho hardiness com as variáveis independentes.

Demonstraram significância com o desfecho hardiness, as seguintes variáveis: ter filhos $(\mathrm{p}=$ $0,050)$, carga horária semanal de 41 a $80 \mathrm{~h}(\mathrm{p}=$ $0,006)$, não tirar licença/afastamento $(\mathrm{p}<0,001)$, estilo de vida fantástico muito bom/excelente ( $\mathrm{p}$ $<0,001)$, atividade física ativa $(\mathrm{p}=0,016)$, possuir dieta balanceada $(\mathrm{p}<0,001)$, bom sono ( $\mathrm{p}$ $<0,001)$, ansiedade leve/ausente $(\mathrm{p}<0,001)$, depressão leve/ausente $(\mathrm{p}<0,001)$, desesperança leve/ausente $(\mathrm{p}<0,001)$, satisfação por compaixão baixa/média $(\mathrm{p}<0,001)$, burnout $(\mathrm{p}<0,001)$, estresse $(\mathrm{p}<0,001)$, autopercepção de saúde excelente/boa $(\mathrm{p}<0,001)$, não fazer uso de medicação para dormir $(\mathrm{p}<0,001)$, não ter problemas autoimunes $(\mathrm{p}=0,031)$, sexuais e reprodutivos ( $\mathrm{p}<0,001)$, mentais $(\mathrm{p}<0,001)$, sanguíneos ( $\mathrm{p}$ $=0,074)$ e transtornos alimentares $(\mathrm{p}=0,035)$.

A análise múltipla na Tabela 4 , evidenciou após o ajuste a significância de cinco variáveis: o estilo de vida fantástico, licença/afastamento do trabalho, satisfação por compaixão, estresse no trabalho e burnout. A chance de alto hardiness entre os profissionais de saúde que possuíam EVF regular ou ruim reduz em $74 \%$ quando comparada aos que possuíam EVF considerado muito bom/excelente. A chance de alto hardiness em profissionais que não tiraram licença/afastamento do trabalho é 3,32 vezes maior, contrastando com aqueles que tiveram histórico de licença/ afastamento.

Profissionais de saúde que apresentavam alta satisfação por compaixão no trabalho possuíam 2,32 vezes mais chances de ter um alto hardiness. A chance de ter alto hardiness em profissionais que possuíam baixo estresse no trabalho foi 2,52 vezes maior quando comparado aos que tinham alto estresse. Profissionais de saúde que apresentaram baixo burnout possuíam 3,06 vezes mais chance de alto hardiness quando comparados com aqueles que possuíam alto esgotamento crônico decorrente do trabalho.

\section{Discussão}

Os resultados deste estudo demonstraram associação entre a personalidade hardiness $e$ determinados fatores tais como: sociodemográficos, ambiente de trabalho, comportamentais, saúde mental e saúde física.

A personalidade hardiness desempenha um papel claro e relevante na diminuição da vulnerabilidade ao estresse, sendo assim, considerada como um fator de proteção para a saúde do trabalhador, pois permite melhorar o desempenho e promover um estilo de vida menos estressante ${ }^{21}$.

Constatou-se neste estudo que a maioria dos profissionais de saúde apresentou classificação dos escores total da EH hardiness moderado/baixo. Dados semelhantes foram verificados em estudo realizado em um hospital em Marrocos no ano de 2018, indicando uma taxa relativamente baixa de resistência do pessoal de saúde, sendo que $81 \%$ dos profissionais apresentaram baixa resistência, 16\% mostraram resistência moderada e apenas $3 \%$ boa resistência ${ }^{22}$.

A associação estatisticamente significativa entre hardiness e características sociodemográficas na resistência psicológica de profissionais de saúde pode ser verificada em outros estudos. Foram identificados como fatores de risco sexo, estado civil, tempo de serviço e horário de trabalho. As mulheres são mais resistentes do que os homens, indivíduos solteiros são mais vulneráveis que os casados e os profissionais com menor tempo de serviço são menos resistentes do que os mais velhos ${ }^{22}$. Entretanto, neste estudo, somente a variável ter filhos $(\mathrm{p}=0,050)$ apresentou a associação com desfecho alto hardiness. Mister, a condução estudos sobre a relação entre as va- 
Tabela 1. Associação da classificação da personalidade hardiness de profissionais de saúde atuantes em serviços hospitalares que atendem pacientes críticos no Norte de Minas Gerais/Brasil, com as variáveis sociodemográficas, $2018(n=469)$.

\begin{tabular}{|c|c|c|c|c|}
\hline \multirow{2}{*}{ Variável } & \multicolumn{3}{|c|}{ Classificação do hardiness no trabalho } & \multirow{2}{*}{ p valor } \\
\hline & Alto $\%$ & Médio\% & Baixo\% & \\
\hline \multicolumn{5}{|l|}{ Sexo } \\
\hline Feminino & 28,2 & 47,1 & 24,7 & 0,714 \\
\hline Masculino & 25,5 & 50,9 & 23,6 & \\
\hline \multicolumn{5}{|l|}{ Faixa etária (anos) } \\
\hline $20-29$ & 25,0 & 44,8 & 30,2 & \\
\hline $30-39$ & 28,8 & 50,2 & 21,0 & 0,718 \\
\hline $40-49$ & 26,7 & 47,7 & 25,6 & \\
\hline$\geq 50$ & 26,7 & 50,0 & 23,7 & \\
\hline \multicolumn{5}{|l|}{ Estado civil } \\
\hline Com companheiro & 29,0 & 49,0 & 22,1 & 0,302 \\
\hline Sem companheiro & 24,6 & 47,5 & 27,9 & \\
\hline \multicolumn{5}{|l|}{ Religião } \\
\hline Não possui & 14,3 & 47,6 & 38,1 & \\
\hline Católica & 27,2 & 50,3 & 22,5 & 0,619 \\
\hline Evangélica & 29,4 & 43,1 & 27,5 & \\
\hline Outras & 33,3 & 46,7 & 20,0 & \\
\hline \multicolumn{5}{|c|}{ Renda familiar (em salários mínimos) } \\
\hline$\leq 3$ & 30,1 & 47,7 & 22,2 & \\
\hline $3-6$ & 27,6 & 45,8 & 26,6 & 0,90 \\
\hline $6-9$ & 21,3 & 59,0 & 19,7 & \\
\hline$>9$ & 25,4 & 47,6 & 27,0 & \\
\hline \multicolumn{5}{|l|}{ Possui filhos } \\
\hline Sim & 27,5 & 51,7 & 20,9 & 0,050 \\
\hline Não & 26,9 & 42,5 & 30,5 & \\
\hline
\end{tabular}

riáveis sociodemográficas, fatores de estresse no trabalho e personalidade resistente pois ainda são $\operatorname{raros}^{23,24}$.

Considerando os fatores relacionados ao ambiente de trabalho, observou-se que houve associação significativa do alto hardiness com as variáveis carga horária semanal de 41 a $80 \mathrm{~h}$ e não tirar licença/afastamento. A carga horária de trabalho é considerada um fator crítico para a resistência ao estresse, uma vez que profissionais de saúde com carga horária extensa haviam desenvolvido baixa resistência à tensão. Enquanto, profissionais que trabalhavam com carga horária média de 40 horas semanais demonstraram maior resistência, sendo considerada como um fator de proteção ${ }^{22}$.

Destarte, os estudos descrevem e reforçam os prejuízos psicológicos que as jornadas de plantão e o excesso de longas horas podem trazer aos profissionais de saúde, sobretudo, aqueles que atendem pacientes críticos, pois a sobrecarga de trabalho foi apontada na literatura como uma das principais fontes de estresse ${ }^{25}$.

Pesquisas afirmam que profissionais com mais tempo de serviço demonstram relação com alto hardiness, visto que desenvolveram estratégia de coping que lhes permitem lidar melhor com as situações estressantes advindas do trabalho ${ }^{26,27}$.

O caminho de transformação de um indivíduo resistente ao enfrentamento envolve querer mudar os eventos de vida estressantes ou pensar sobre eles de forma otimista. Outra ação complementar é a melhoria das práticas de saúde pessoais, consequência de suas crenças sobre os próprios comportamentos de saúde ${ }^{28}$.

Neste estudo foi possível verificar associação entre alto hardiness e profissionais que possuíam Estilo de Vida Fantástico muito bom/excelente. Corroborando estes resultados, estudos afirmam que, em geral, resistência tende a ser associada com melhores comportamentos relacionados à saúde, tais como a manutenção de um regime de exer- 
Tabela 2. Associação da classificação da personalidade hardiness de profissionais de saúde atuantes em serviços hospitalares que atendem pacientes críticos no Norte de Minas Gerais/Brasil, com as variáveis saúde física e mental, $2018(\mathrm{n}=469)$.

\begin{tabular}{|c|c|c|c|c|}
\hline \multirow[t]{2}{*}{ Variável } & \multicolumn{3}{|c|}{$\begin{array}{c}\text { Classificação } \\
\text { do hardiness no } \\
\text { trabalho }\end{array}$} & \multirow[t]{2}{*}{ p valor } \\
\hline & Alto & Médio & Baixo & \\
\hline \multicolumn{5}{|c|}{ Problemas de saúde (físico) } \\
\hline \multicolumn{5}{|l|}{ Cardíacos } \\
\hline Não & 126 & 214 & 109 & 0,175 \\
\hline Sim & 2 & 13 & 5 & \\
\hline \multicolumn{5}{|l|}{ Sanguíneos } \\
\hline Não & 125 & 216 & 104 & 0,074 \\
\hline Sim & 13 & 11 & 10 & \\
\hline \multicolumn{5}{|l|}{ Autoimunes } \\
\hline Não & 114 & 198 & 89 & 0,031 \\
\hline Sim & 14 & 29 & 25 & \\
\hline \multicolumn{5}{|l|}{ Endócrinos } \\
\hline Não & 118 & 214 & 105 & 0,660 \\
\hline Sim & 10 & 13 & 9 & \\
\hline \multicolumn{5}{|l|}{ Ósseos } \\
\hline Não & 109 & 181 & 90 & 0,370 \\
\hline Sim & 19 & 46 & 24 & \\
\hline \multicolumn{5}{|l|}{ Digestivos } \\
\hline Não & 114 & 186 & 95 & 0,200 \\
\hline Sim & 14 & 41 & 19 & \\
\hline \multicolumn{5}{|l|}{ Renais/Urinários } \\
\hline Não & 116 & 212 & 105 & 0,639 \\
\hline Sim & 12 & 15 & 9 & \\
\hline \multicolumn{5}{|l|}{ Hepáticos } \\
\hline Não & 126 & 221 & 110 & 0,629 \\
\hline Sim & 2 & 6 & 4 & \\
\hline \multicolumn{5}{|l|}{ Respiratórios } \\
\hline Não & 124 & 218 & 106 & 0,300 \\
\hline Sim & 4 & 9 & 8 & \\
\hline \multicolumn{5}{|c|}{ Reprodutivos/Sexuais } \\
\hline Não & 119 & 208 & 89 & $<0,001$ \\
\hline Sim & 9 & 19 & 25 & \\
\hline \multicolumn{5}{|l|}{ Mentais } \\
\hline Não & 120 & 190 & 77 & $<0,001$ \\
\hline Sim & 8 & 37 & 37 & \\
\hline \multicolumn{5}{|c|}{ Transtornos alimentares } \\
\hline Não & 128 & 221 & 108 & 0,035 \\
\hline Sim & 0 & 6 & 6 & \\
\hline \multicolumn{5}{|l|}{ Oculares/Visuais } \\
\hline Não & 96 & 164 & 78 & 0,521 \\
\hline Sim & 32 & 63 & 36 & \\
\hline \multicolumn{5}{|l|}{ Neurológicos } \\
\hline Não & 119 & 204 & 100 & 0,381 \\
\hline Sim & 9 & 23 & 14 & \\
\hline
\end{tabular}

Tabela 2. Associação da classificação da personalidade hardiness de profissionais de saúde atuantes em serviços hospitalares que atendem pacientes críticos no Norte de Minas Gerais/Brasil, com as variáveis saúde física e mental, $2018(\mathrm{n}=469)$.

\begin{tabular}{|c|c|c|c|c|}
\hline \multirow[t]{2}{*}{ Variável } & \multicolumn{3}{|c|}{$\begin{array}{c}\text { Classificação } \\
\text { do hardiness no } \\
\text { trabalho }\end{array}$} & \multirow[t]{2}{*}{ p valor } \\
\hline & Alto & Médio & Baixo & \\
\hline \multicolumn{5}{|l|}{ Auditivos } \\
\hline Não & 124 & 211 & 102 & 0,073 \\
\hline Sim & 4 & 16 & 12 & \\
\hline \multicolumn{5}{|l|}{ Tumores malignos } \\
\hline Não & 127 & 226 & 113 & 0,868 \\
\hline $\operatorname{Sim}$ & 1 & 1 & 1 & \\
\hline \multicolumn{5}{|l|}{ Dor aguda } \\
\hline Não & 117 & 204 & 102 & 0,858 \\
\hline $\operatorname{Sim}$ & 11 & 23 & 12 & \\
\hline \multicolumn{5}{|l|}{ Dor crônica } \\
\hline Não & 120 & 208 & 104 & 0,716 \\
\hline Sim & 8 & 19 & 10 & \\
\hline \multicolumn{5}{|l|}{ Problemas de saúde (mental) } \\
\hline \multicolumn{5}{|l|}{ Ansiedade } \\
\hline Ausente/Leve & 122 & 210 & 87 & $<0,001$ \\
\hline Moderada/Grave & 6 & 17 & 27 & \\
\hline \multicolumn{5}{|l|}{ Depressão } \\
\hline Ausente/Leve & 128 & 223 & 99 & $<0,001$ \\
\hline Moderada/Grave & 0 & 4 & 15 & \\
\hline \multicolumn{5}{|l|}{ Desesperança } \\
\hline Ausente/Leve & 128 & 223 & 105 & $<0,001$ \\
\hline Moderada/Grave & 0 & 4 & 9 & \\
\hline \multicolumn{5}{|l|}{ Satisfação por compaixão } \\
\hline Baixa/Média & 35 & 122 & 66 & $<0,001$ \\
\hline Alta & 93 & 105 & 28 & \\
\hline \multicolumn{5}{|l|}{ Aparência corporal } \\
\hline Muito satisfeito/Satisfeito & 79 & 142 & 59 & \\
\hline Indiferente & 7 & 23 & 14 & 0,142 \\
\hline $\begin{array}{l}\text { Insatisfeito/Muito } \\
\text { insatisfeito }\end{array}$ & 42 & 62 & 41 & \\
\hline \multicolumn{5}{|l|}{$\begin{array}{l}\text { Uso de medicamentos } \\
\text { psicotrópicos }\end{array}$} \\
\hline Nunca & 120 & 182 & 80 & $<0,001$ \\
\hline Sim & 11 & 27 & 12 & \\
\hline \multicolumn{5}{|l|}{ Autopercepção da saúde } \\
\hline Excelente/Boa & 112 & 187 & 76 & $<0,001$ \\
\hline $\begin{array}{l}\text { Regular/Ruim/Muito } \\
\text { ruim }\end{array}$ & 16 & 40 & 38 & \\
\hline \multicolumn{5}{|l|}{$\begin{array}{l}\text { Estresse traumático } \\
\text { secundário }\end{array}$} \\
\hline Baixo & 107 & 137 & 39 & $<0,001$ \\
\hline Médio/ Alto & 21 & 90 & 75 & \\
\hline \multicolumn{5}{|l|}{ Burnout } \\
\hline Baixo & 120 & 180 & 44 & $<0,001$ \\
\hline Médio & 8 & 47 & 70 & \\
\hline
\end{tabular}


Tabela 3. Associação da classificação da personalidade hardiness de profissionais de saúde atuantes em serviços hospitalares que atendem pacientes críticos no norte de Minas Gerais/Brasil, com as variáveis relacionadas a ambiente de trabalho e comportamento, $2018(n=469)$.

\begin{tabular}{|c|c|c|c|c|}
\hline \multirow{2}{*}{ Variável } & \multicolumn{3}{|c|}{ Classificação do hardiness no trabalho } & \multirow{2}{*}{ p valor } \\
\hline & Alto & Médio & Baixo & \\
\hline \multicolumn{5}{|l|}{ Ambiente de trabalho } \\
\hline \multicolumn{5}{|l|}{ Setor } \\
\hline Pronto Socorro & 43 & 78 & 27 & \\
\hline Nefrologia & 40 & 70 & 38 & 0,370 \\
\hline Oncologia & 27 & 46 & 34 & \\
\hline Terapia Intensiva Neonatal & 18 & 33 & 15 & \\
\hline \multicolumn{5}{|l|}{ Carga horária } \\
\hline Menos de 40 & 20 & 49 & 37 & \\
\hline $41-80$ & 106 & 164 & 72 & 0,006 \\
\hline$>80$ & 2 & 13 & 5 & \\
\hline \multicolumn{5}{|l|}{ Turno de trabalho } \\
\hline Diurno & 67 & 123 & 64 & 0,712 \\
\hline Noturno & 38 & 57 & 25 & \\
\hline Diurno/Noturno & 23 & 47 & 25 & \\
\hline \multicolumn{5}{|c|}{ Tempo de trabalho na área da saúde (anos) } \\
\hline$<5$ & 27 & 45 & 26 & \\
\hline $5-10$ & 38 & 73 & 39 & \\
\hline $10-15$ & 33 & 55 & 26 & 0,975 \\
\hline $15-20$ & 14 & 30 & 11 & \\
\hline \multicolumn{5}{|l|}{ Tempo de trabalho no setor (anos) } \\
\hline$<5$ & 77 & 118 & 54 & \\
\hline $5-10$ & 29 & 66 & 41 & 0,206 \\
\hline$>10$ & 22 & 43 & 19 & \\
\hline \multicolumn{5}{|l|}{ Múltiplos vínculos de trabalho } \\
\hline Apenas um emprego & 87 & 138 & 71 & \\
\hline Dois empregos & 35 & 71 & 36 & 0,638 \\
\hline Três ou mais empregos & 6 & 18 & 7 & \\
\hline \multicolumn{5}{|l|}{ Licenças/ Afastamento do trabalho } \\
\hline Não & 123 & 205 & 92 & $<0,001$ \\
\hline Sim & 5 & 22 & 22 & \\
\hline \multicolumn{5}{|l|}{ Características comportamentais } \\
\hline \multicolumn{5}{|l|}{ Estilo de vida fantástico } \\
\hline Regular/ Necessita melhorar & 2 & 11 & 18 & \\
\hline Bom & 10 & 61 & 53 & \\
\hline Muito bom/ Excelente & 116 & 155 & 43 & $<0,001$ \\
\hline \multicolumn{5}{|l|}{ Tabagismo } \\
\hline Não & 128 & 219 & 110 & 0,099 \\
\hline Sim & 0 & 8 & 4 & \\
\hline \multicolumn{5}{|l|}{ Bom padrão de sono } \\
\hline Não & 36 & 101 & 70 & \\
\hline Sim & 92 & 126 & 24 & $<0,001$ \\
\hline \multicolumn{5}{|l|}{ Prática de atividade física } \\
\hline Não & 75 & 142 & 86 & 0,016 \\
\hline Sim & 53 & 85 & 28 & \\
\hline \multicolumn{5}{|l|}{ Alimentação balanceada } \\
\hline Não & 42 & 93 & 64 & \\
\hline Sim & 86 & 134 & 50 & \\
\hline \multicolumn{5}{|l|}{ Peso ideal } \\
\hline Não & 88 & 165 & 78 & 0,623 \\
\hline Sim & 40 & 62 & 36 & \\
\hline
\end{tabular}


Tabela 4. Regressão logística da relação entre alto hardiness e variáveis estilo de vida fantástico (EVF), licença/ afastamento do trabalho, satisfação por compaixão no trabalho, estresse no trabalho (ETT) e burnout em profissionais de saúde atuantes em serviços hospitalares que atendem pacientes críticos no Norte de Minas Gerais/ Brasil, $2018(\mathrm{n}=469)$.

\begin{tabular}{|c|c|c|c|}
\hline \multirow[t]{2}{*}{ Variável } & \multicolumn{2}{|c|}{$\begin{array}{l}\text { Classificação } \\
\text { altohardiness }\end{array}$} & \multirow[t]{2}{*}{ p valor } \\
\hline & OR & IC & \\
\hline \multicolumn{4}{|l|}{ EV Fantástico } \\
\hline $\begin{array}{l}\text { Muito bom/ } \\
\text { Excelente }\end{array}$ & 1,00 & $(0,08-1,63)$ & \\
\hline Bom & 0,35 & $(0,13-0,53)$ & 0,181 \\
\hline Regular/ Ruim & 0,26 & & $<0,001^{\star}$ \\
\hline \multicolumn{4}{|l|}{ Licença } \\
\hline Sim & 1,00 & & \\
\hline Não & 3,32 & $(1,20-9,16)$ & 0,021 \\
\hline \multicolumn{4}{|l|}{ Satisfação por } \\
\hline \multicolumn{4}{|l|}{ Compaixão } \\
\hline Baixo/ Médio & 1,00 & & \\
\hline Alto & 2,32 & $(1,42-3,79)$ & 0,001 \\
\hline \multicolumn{4}{|l|}{ Estresse } \\
\hline Médio/Alto & 1,00 & & \\
\hline Baixo & 2,52 & $(1,58-4,00)$ & $<0,001$ \\
\hline \multicolumn{4}{|l|}{ Burnout } \\
\hline Médio & 1,00 & & \\
\hline Baixo & 3,06 & $(1,35-6,92)$ & 0,007 \\
\hline
\end{tabular}

cício regular, ingestão nutricional adequada e ter uma abordagem equilibrada para o trabalho e vida $^{29-31}$.

Destaca-se ainda que a personalidade hardiness é apontada na adaptação ou ajustamento de doenças, indicando uma forte associação entre adoecer e resistência psicológica. Dentre as variáveis físicas analisadas, apresentaram associação estatística: a ausência de problemas autoimunes, problemas sexuais e reprodutivos, mentais, sanguíneos e transtornos alimentares. No entanto, a significância da associação não se confirmou após o ajuste no modelo múltiplo. Resultados semelhantes também foram constatados em outro estudo que problemas físicos não permaneceram significantes ${ }^{22}$.

Não obstante, a resistência é considerada pela maioria dos pesquisadores como uma construção relevante para o estudo da saúde, e há um consenso sobre os efeitos protetores de resistência na saúde física, Brooks ${ }^{32}$ apresentou uma revisão abrangente de tais estudos e resultados. Todavia, a forma como ela exerce esta influência ainda não foi suficientemente estabelecida.

Em relação à saúde mental, foi possível verificar a associação entre personalidade hardiness com níveis leve/ausente de ansiedade, depressão, desesperança, satisfação por compaixão (SC), burnout, estresse e autopercepção de saúde excelente/boa.

Vários estudos correlacionam negativamente os sintomas psicossomáticos com dimensões da resistência psicológica. Os desfechos indicam que a resistência pode servir como um tampão contra o estresse e é um fator de proteção. Em outro estudo, os resultados ligam hardiness à saúde mental e física e ainda sugerem que a saúde mental conota um caminho pelo qual hardiness influencia a saúde física ${ }^{33,34}$.

Pesquisa realizada com estudantes de cursos técnicos em enfermagem constatou a existência de correlação negativa de baixa intensidade e estatisticamente significante entre BDI versus Compromisso, Controle e Desafio, o que possibilita inferir que aqueles alunos que apresentam características hardy não apresentam sintomas depressivos ${ }^{35}$.

A associação entre hardiness e desesperança pode ser ratificada em estudo realizado com enfermeiros iranianos evidenciando que o hardiness é um fator de proteção contra o estresse percebido e um fator facilitador para a felicidade em enfermeiros. Ademais, profissionais com baixos níveis de estresse eram mais propensos a relatar maior resistência e felicidade ${ }^{36}$.

Ressalta-se que estudo em uma amostra de enfermeiros na Espanha, sobre capacidade preditiva da personalidade resistente e a auto eficácia generalizada sobre a percepção do estado geral de saúde, revelou-se estatisticamente relacionada e em um sentido positivo ${ }^{37}$. Essa influência provavelmente ocorre via múltiplos aspectos da saúde mental, como sofrimento subjetivo, enfrentamento/avaliação, burnout, práticas saudáveis e diferenças hormonais relacionadas ao estresse ${ }^{34}$.

A análise multivariável evidenciou aumento na chance de desenvolver alto hardiness nos profissionais dos serviços de saúde abordado que apresentavam alta satisfação por compaixão (SC) ( $<$ 0,001, OR 2,32 [1-42-3,79]); baixo burnout ( $\mathrm{p}<0,0007$, OR 3,03 [1,35-6,92]) e baixo estresse no trabalho $(\mathrm{p}<0,001$, OR $2,52[1,58-4,00])$.

Estudos apontam a relação entre o baixo nível de resistência e a sua extensão com os problemas relacionados à saúde mental e seus componentes. Sendo assim, personalidade resistente 
ameniza a chance de desenvolver o burnout. Os resultados evidenciaram que resistência explica $35 \%$ da variância do burnout em uma amostra de enfermeiros. E, mais especificamente, o mesmo grupo de pesquisa confirmou os efeitos positivos sobre a maior resistência ao estresse na equipe de enfermagem ${ }^{28-31,34}$.

Dessa forma, percebe-se que a personalidade hardiness tem sido analisada com uma estratégia de fortalecimento de outras características pessoais, como o desempenho, autoeficácia, senso de controle e de redução dos efeitos do estresse, reforçando a assertiva de que hardiness facilita ações que modificam a interpretação das circunstâncias estressantes com manutenção ou até aumento da saúde dos indivíduos frente aos estressores e seus efeitos ${ }^{38}$.

Considerando a relevância da personalidade hardiness na promoção à saúde e estilo de vida saudável, deve-se orientar os serviços de saúde para que instituam programas em que a resistência possa ser desenvolvida e, consequentemente, reduzida a probabilidade de estar envolvido com doenças $^{4,5,9,39}$.

Para a instituição de saúde, os benefícios de ter pessoas hardy em seu quadro de colaboradores permeiam a eficácia e eficiência, a qualidade dos serviços prestados, a diminuição de custos com afastamento e licenças médicas, a redução do absenteísmo e rotatividade de pessoal ${ }^{40}$.

\section{Conclusão}

Os resultados deste estudo demonstraram que a personalidade hardiness influência direta e indiretamente a saúde e bem-estar, promovendo o uso de recursos sociais e alterando o estresse auto percebido, e reduzindo assim a tensão no trabalho em saúde.

O bem-estar mental e físico também foram relacionados à presença de personalidade resistente. A chance de ter alto hardiness foi associada à ausência de licença/afastamento do trabalho, alta satisfação por compaixão no trabalho, baixo estresse no trabalho e baixo burnout, corroborando os achados da literatura que indicam a presença de personalidade hardiness como fator de proteção para a saúde. Ademais, fazem-se necessários estudos que esclareçam a relação entre a personalidade hardiness e fatores socioeconômicos como gênero, idade, renda e a influência do mesmo na gênese das doenças físicas.

Impõe-se como necessário e desafiador, desenvolver a resistência psicológica nos profissionais de saúde, sobretudo nos serviços que atendem pacientes críticos, a fim de se manter e melhorar a sua capacidade de manter o bem-estar pessoal e profissional, facilitando o coping transformacional em face do contínuo estresse no trabalho e na adversidade.

\section{Colaboradores}

ECS Alves, RF Silva-Junior, SP Santos, HA Barbosa, JDPRV Torres e CSO Silva conceberam, desenharam, executaram, analisaram e interpretaram os dados do estudo. KO Santos e LG Siqueira contribuíram nas análises e interpretação dos dados. Todos os autores redigiram e participaram da revisão final do manuscrito. 


\section{Referências}

1. Jacques JPB, Oussak LCS, Scholze AR, Ribeiro BGA, Martins JT, Perfeito RR. Personalidade hardiness ecoping entre profissionais de enfermagem do centro cirúrgico. Rev enferm UFPE online[periódico na Internet]. 2017 Nov [acessado 2018 Out 10]; 11(11):[cerca de 7 p.]. Disponível em: https://periodicos.ufpe.br/revistas/revistaenfermagem/article/ download/231203/25202

2. Ferreira CAA, Reis Neto MT, Kilimnik ZM, Santos AS. O Contexto do Estresse Ocupacional dos Trabalhadores da Saúde: estudo bibliométrico. Revista de Gestão em Sistemas de Saúde - RGSSline [periódico na Internet]. 2016 Jul-Dez [acessado 2018 Out 10]; 5(2):[cerca de 16 p.]. Disponível em: http://www. revistargss.org.br/ojs/index.php/rgss/article/download/233/188

3. Maturana APPM, Valle TGMV. Estratégias de enfrentamento e situações estressoras de profissionais no ambiente hospitalar. Psicol Hosp [periódico na Internet]. 2014 [acessado 2018 set 10]; 12 (2): [cerca de 22 p.]. Disponível em: http://pepsic.bvsalud.org/pdf/ph/ v12n2/12n2a02.pdf

4. Maddi SR. The story of hardiness: twenty years of theorizing, research, and practice. Consult Psychol J 2002; 54(1):173-185.

5. Kobasa SC. Eventos estressantes de vida, personalidade e saúde: Um inquérito na robustez. J Pers Soc Psychol 1979; 7(1):1-11.

6. Vieira HP. Estresse ocupacional, sindrome de Burnout $e$ hardiness em professores de colégio militar [dissertação]. Campo Grande: Universidade Católica Dom Bosco; 2007.

7. Kobasa SC, Maddi SR, Kahn S. Rusticidade e saúde: estudo prospectivo. J Pers Soc Psychol 1982; 42(1):168177.

8. Kobasa SC, Maddi SR, Courington S. Personality and constitution as mediators in the stressillness relationship. J Health Soc Behav 1981; 22(4):368-378.

9. Maddi SR. Questões e intervenções em estresse Mastery. Em: Friedman SH, organizador. Personalidade e doença. New York: John Wiley \& Sons; 1990. p. 121154.

10. Farooqi YN. Rusticidade e Lidar como preditores de Professional Estresse vida entre médicos Casa-trabalho. EUA: University; 2015.

11. Hague HRN, Leggat GL. Enhancing hardiness among health-care workers: the perceptions of senior managers. Health Services Management Research 2010; 23:54-59.

12. Jalali S, Amarqan HA. Estudo da relação entre resistência psicológica e criatividade com o estresse do trabalho de pessoal dos serviços sociais de emergência de Golestan. Indian Journal of Fundamental and Applied Life Sciences 2015; 5(2):1671-1679.

13. Minas Gerais. Secretaria de Estado de Saúde (SES). Plano Diretor de Regionalização. Adscrição e população dos municípios por macrorregiões e microrregiões de saúde. Belo Horizonte: SES; 2016.
14. Serrano PM, Bianchi ERF. Validação da Escala de Hardiness (HS):confiabilidade e validade de construto. J Health Sci Inst [periódico na Internet]. 2013 July/ Sept [acessado 2018 Out 10]; 31(3):[cerca de 10 p.]. Disponível em: https://www.unip.br/presencial/comunicacao/publicacoes/ics/edicoes/2013/03_jul-set/ V31_n3_2013_p292a295.pdf

15. Añez CRR, Reis RS, Petroski EL. Versão brasileira do questionário "estilo de vida fantástico". Arq Bras Cardiol 2008; 91(2):102-109.

16. Fleiss JL, Levin B, Paik M. Statistical methods for rates and proportions. New Jersey: John Wiley \& Sons; 2003.

17. Stamm BH. The Concise ProQOL Manual. Pocatello: ProQOL.org; 2010.

18. Paschoal T, Tamayo A. Validação da Escala de Estresse no trabalho. Estudos de Psicologia [periódico na Internet]. 2004 [acessado 2013 Out 10]; 9(1):[cerca de 10 p.]. Disponível em: http://www.scielo.br/pdf/epsic/ v9n1/22380.pdf

19. Vicente CS, Oliveira RA, Maroco J. Análise Fatorial do Inventário de Burnout de Maslach (MBI-HSS) em profissionais portugueses. Psic., Saúde \& Doenças [periódico na Internet]. 2013 Mar [acessado 2018 Dez 10]; 14(1):[cerca de 10 p.]. Disponível em: http:// www.scielo.mec.pt/pdf/psd/v14n1/v14n1a10.pdf

20. Cunha JA. Manual da versão em português das escalas Beck. Tradução e adaptação brasileira. Belo Horizonte: Casa do Psicólogo; 2001.

21. Freitas FMB de, Vannuchi MTO, Haddad MCL, Silva LGC, Rossaneis MA. Hardiness e estresse ocupacional em enfermeiros gestores de instituições hospitalares. Ver Enferm UFPE online[periódico na Internet]. 2017 Out, [acessado 2018 Dez 10]; 11(Supl. 10):[cerca de 7 p.]. Disponível em: https://periodicos.ufpe.br/revistas/revistaenfermagem/article/viewFile/231183/25162

22. Chtibi, H., Ahami, A., Azzaoui, FZ, Khadmaoui, A. Mammad, K. e Elmassioui, F. Study of Psychological Resilience among Health Care Professionals, in IbnSina Hospital/Rabat/Morocco. Int J Clin Saúde Psychol[periódico na Internet]. 2018 Jul [acessado 2018 Dez 10]; 7(3):[cerca de 7 p.]. Disponível em: https:// file.scirp.org/pdf/OJMP_2018070915020071.pdf

23. Sansone C, Wiebe DJ, Morgan C. Self-regulating interest: the moderating role of hardiness and conscientiousness. J Pers 1999; 67(4):701-733.

24. Nezhad MAS, Besharat MA. Relations of resilience and hardiness with sport achievement and mental health in a sample of athletes. Procedia Soc Behav Sci 2010; 5(1):757-763.

25. Rincón LB, Guarino L. Estrés laboral, afrontamento, sensibilidad emocional y síntomas físicos y psicológicos em médicos venezolanos. Revista Colombiana de Psicologia-RCP 2008; 17(1):43.

26. Saksvik-Lehouillier I, Bjorvatn B, Magerøy, N Pallesen S. Hardiness, psychosocial factors and shift work tolerance amongnurses a 2 year follow-up study. $J$ Ad$v$ Nurs-Wiley Online Library[periódico na Internet]. 2016 [acessado 2019 Jan 10]; 59(1):[cerca de 13 p.]. Disponível em: https://onlinelibrary.wiley.com/doi/ epdf/10.1111/jan.12951 
27. Souza WC, Silva AMM. A influência de fatores de personalidade e de organização do trabalho no burnout em profissionais de saúde. Estudos de Psicologia (Campinas) [periódico na Internet]. 2002 [acessado 2018 Out 10], 19(1):[cerca de 12 p.]. Disponível em: https://dx.doi.org/10.1590/S0103-166X 2002000100004

28. Garrosa E, Moreno-Jiménez B, Liangb Y, González JL. The relationship between socio-demographic variables, job stressors, burnout, and hardy personality in nurses: an exploratory study. Int J Stud Stud [periódico na Internet]. 2006 [acessado 2018 Dez 10], 45(3):[cerca de 12 p.]. Disponível em: https://doi. org/10.1016/j.ijnurstu.2006.09.003

29. Brehm B. Management: increasing your stress resistance. New York: Longman; 1998.

30. Bigbee JL. Hardiness: a new perspective in health promotion. Nurse Pract 1985; 10(11):51-56.

31. Rossi E. The Break 20 minutes: reduce stress, maximize performance and improve health and emotional well-being using the new of science ultradian rhythms. Los Angeles: JP Tarcher; 1991.

32. Brooks MV. Health-related hardiness and chronic illness: asynthesis of current research. Nursing Forum 2003; 38(3):11-20.

33. Moreno-Jiménez B, Rodríguez-Muñoz A, Hernández EG, Blanco LM. Desenvolvimento e validação da Ocupacional Hardiness. Questionnaire Psicothema 2014; 26(2):207-214

34. Taylor MK, Pietrobon R, Taverniers J, Leon MR, Fern BJ. Relationships of hardiness to physical and mental health status in military men: a test of mediated effects. J Behav Med 2011; 36(1):1-9.

35. Grazziano ES, Bianchini C, Lopes LFD, Souza BF, Franco DM. Resistência ao estresse e depressão em estudantes de cursos técnicos em enfermagem. Rev enferm UFPE on line 2015; 9(Supl. 2):837-843.

36. Abdollahi A, Abu Talib H, Yaacob SN, Ismail Z. Hardiness as a mediator between perceived stress and happiness in nurses. J PsychiatrMent Health Nurs [periódico na Internet. 2014 [acessado 2019 Jan 10]; 21(9):[cerca de 8 p.]. Disponível em: https://doi. org/10.1111/jpm.12142

37. Rísquez, MIR,Meca JS, Fernández CG.Personalidad resistente, autoeficacia y estado general de salud en profesionales de enfermería de cuidados intensivos y urgencias. Psicothema 2010; 22(4):600-605.

38. Silva RM, Goulart CT, Bolzan MEO, Serrano PM, Lopes LFD, Guido, LA. Estresse e hardiness em residentes médicos. Rev enferm UFPE on line 2013; 7(9):5406-5413.

39. Maddi SR. Comentários em Trends in Hardiness Investigação e Teorizando. Science Psychology Journal 1999; 51(2):67-71.

40. Batista KM, Silva ROC, Grazziano ES. Personalidade resistente nas equipes médica e de enfermagem em centro cirúrgico. Rev. SOBECC 2014; 19(4):214-218. 
